\title{
mau \\ On the Nonlocal Problems in Time for Time-Fractional Subdiffusion Equations
}

\author{
Ravshan Ashurov ${ }^{1, *,+}$ (1) and Yusuf Fayziev ${ }^{2,+}$ \\ 1 Institute of Mathematics, Uzbekistan Academy of Science, Student Town Str., Tashkent 100174, Uzbekistan \\ 2 University of Uzbekistan Named after Mirzo Ulugbek, Student Town Str., Tashkent 100174, Uzbekistan; \\ fayziev.yusuf@mail.ru \\ * Correspondence: ashurovr@gmail.com; Tel.: +998-903470011 \\ + These authors contributed equally to this work.
}

check for updates

Citation: Ashurov, R.; Fayziev, Y. On the Nonlocal Problems in Time for

Time-Fractional Subdiffusion

Equations. Fractal Fract. 2022, 6, 41 https://doi.org/10.3390/

fractalfract6010041

Academic Editor: Maria Rosaria Lancia

Received: 22 November 2021

Accepted: 29 December 2021

Published: 12 January 2022

Publisher's Note: MDPI stays neutral with regard to jurisdictional claims in published maps and institutional affiliations.

Copyright: (C) 2022 by the authors. Licensee MDPI, Basel, Switzerland. This article is an open access article distributed under the terms and conditions of the Creative Commons Attribution (CC BY) license (https:// creativecommons.org/licenses/by/ $4.0 /)$.
Abstract: The nonlocal boundary value problem, $d_{t}^{\rho} u(t)+A u(t)=f(t)(0<\rho<1,0<t \leq T)$, $u(\xi)=\alpha u(0)+\varphi(\alpha$ is a constant and $0<\xi \leq T)$, in an arbitrary separable Hilbert space $\mathrm{H}$ with the strongly positive selfadjoint operator $\mathrm{A}$, is considered. The operator $d_{t}$ on the left hand side of the equation expresses either the Caputo derivative or the Riemann-Liouville derivative; naturally, in the case of the Riemann-Liouville derivatives, the nonlocal boundary condition should be slightly changed. Existence and uniqueness theorems for solutions of the problems under consideration are proved. The influence of the constant $\alpha$ on the existence of a solution to problems is investigated. Inequalities of coercivity type are obtained and it is shown that these inequalities differ depending on the considered type of fractional derivatives. The inverse problems of determining the right-hand side of the equation and the function $\varphi$ in the boundary conditions are investigated.

Keywords: nonlocal problems; the Riemann-Liouville and the Caputo derivatives; subdiffusion equation; inverse problems

MSC: Primary 35R11; Secondary 34A12

\section{Introduction}

Let $H$ be a separable Hilbert space with the scalar product $(\cdot, \cdot)$ and the norm $\|\cdot\|$ and $A: H \rightarrow H$ be an arbitrary unbounded positive selfadjoint operator in $H$. Suppose that $A$ has a complete in $H$ system of orthonormal eigenfunctions $\left\{v_{k}\right\}$ and a countable set of positive eigenvalues $\lambda_{k}$. It is convenient to assume that the eigenvalues do not decrease as their number increases, i.e., $0<\lambda_{1} \leq \lambda_{2} \cdots \rightarrow+\infty$.

Using the definitions of a strong integral and a strong derivative, fractional analogues of integrals and derivatives can be determined for vector-valued functions (or simply functions) $h: \mathbb{R}_{+} \rightarrow H$, while the well-known formulae and properties are preserved (see, e.g., [1]). Recall that the fractional integration of order $\sigma<0$ of the function $h(t)$ defined on $[0, \infty)$ has the form

$$
\partial_{t}^{\sigma} h(t)=\frac{1}{\Gamma(-\sigma)} \int_{0}^{t} \frac{h(\xi)}{(t-\xi)^{\sigma+1}} d \xi, \quad t>0,
$$

provided the right-hand side exists. Here $\Gamma(\sigma)$ is Euler's gamma function. Using this definition one can define the Riemann-Liouville fractional derivative of order $\rho, 0<\rho<1$, as

$$
\partial_{t}^{\rho} h(t)=\frac{d}{d t} \partial_{t}^{\rho-1} h(t) .
$$


If in this definition we interchange differentiation and fractional integration, then we obtain the definition of the regularized derivative, that is, the definition of the fractional derivative in the sense of Caputo:

$$
D_{t}^{\rho} h(t)=\partial_{t}^{\rho-1} \frac{d}{d t} h(t)
$$

Note that if $\rho=1$, then fractional derivatives coincides with the ordinary classical derivative of the first order: $\partial_{t} h(t)=D_{t} h(t)=\frac{d}{d t} h(t)$.

Let $\rho \in(0,1)$ be a fixed number and let $C((a, b) ; H)$ stand for a set of continuous functions $u(t)$ of $t \in(a, b)$ with values in $H$.

The subject of this work is the following two nonlocal boundary value problems:

$$
\left\{\begin{array}{l}
D_{t}^{\rho} u(t)+A u(t)=f(t), \quad 0<t \leq T \\
u(\xi)=\alpha u(0)+\varphi, \quad 0<\xi \leq T
\end{array}\right.
$$

and

$$
\left\{\begin{array}{l}
\partial_{t}^{\rho} u(t)+A u(t)=g(t), \quad 0<t \leq T \\
\left.\partial_{t}^{\rho-1} u(t)\right|_{t=\xi}=\alpha \lim _{t \rightarrow 0} \partial_{t}^{\rho-1} u(t)+\phi, \quad 0<\xi \leq T,
\end{array}\right.
$$

where $f(t), g(t) \in C((0, T] ; H), \varphi, \phi \in H$ and $\alpha$ is a constant, $\xi$-fixed point. These problems are also called the forward problems.

Definition 1. A function $u(t) \in C([0, T] ; H)$ with the properties $D_{t}^{\rho} u(t), A u(t) \in C((0, T) ; H)$ and satisfying conditions (2) is called the solution of the nonlocal problem (2).

The definition of the solution to the nonlocal problem (3) is introduced in a similar way.

If $\alpha=0$ (and $\xi=T$ ), then these problems are called the backward problems. The backward problems in case (2) were studied in detail, for example, in [2-4]. The work [5] is devoted to the study of the backward problem in case (3). Therefore, in what follows we only consider the case

$$
\alpha \neq 0 .
$$

The backward problems for the diffusion process are of great importance in engineering fields and are aimed at determining the previous state of a physical field (for example, at $t=0$ ) based on its current information (see, e.g., [3] and for the classical head equation see [6]). However, regardless of the fact that the Riemann-Liouville or the Caputo derivative is taken into the equation, this problem is ill-possed in the sense of Hadamard. In other words, a small change of $u(T)$ in the norm of space $H$ leads to large changes in the initial data. As can be seen from the main results of papers [2-5] (note, in these works $0<\rho<1$ ), the situation changes if we take the sufficiently smooth function $u(T)$. Since the problem is ill-posed, many authors have considered various regularization options for finding the initial condition (see, for one-dimensional elliptical part, Liu and Yamamoto [2], for the nonlinear case, Tuan, Huynh, Ngoc, and Zhou [7]). In particular, as for numerical approaches, see Tuan, Long and Tatar [8], Wang and Liu [9] and the references therein.

In the case $\rho=1$ these problems are also called (see, e.g., [6], p. 214) the inverse heat conduction problem with inverse time (retrospective inverse problem). It should also be noted that, in this case, even the smoothness of the function $u(T)$ does not guarantee the stability of the solution (see, e.g., Chapter 8.2 of [6]).

As we know, in most models described by differential (and pseudodifferential, see e.g., [10]) equations the initial condition is used. However, in practice, some other models have to use nonlocal conditions, for example, including integrals over time intervals (see, e.g., [11] for reaction-diffusion equations or [12] for fractional equations), or connecting the solution at different times, for instance at the initial time and at the terminal time (see, e.g., $[13,14]$ ). 
Note, nonlocal conditions express and explain some full details about natural events because they consider additional information in the initial conditions.

The following nonlocal boundary value problem for the classical diffusion equation

$$
\left\{\begin{array}{l}
u^{\prime}(t)+A u(t)=f(t), \quad 0<t \leq T ; \\
u(\xi)=u(0)+\varphi, \quad 0<\xi \leq T
\end{array}\right.
$$

in an arbitrary Banach space $E$ with the strongly positive operator $A$, has been extensively studied by numerous researchers (see, e.g., A. O. Ashyralyev et al. [13,14]). As shown in these papers, in contrast to the retrospective inverse problem, the problem (5) is coercively solvable in some spaces of differentiable functions. It should also be noted that various nonlocal boundary value problems for parabolic equations reduce to the boundary value problem (5) (see, e.g., [15], Chapter 1).

In the present paper we prove the existence and uniqueness theorems for solutions of problems (2) and (3). Next, we will study the dependence of the existence of a solution on the value of the parameter $\alpha$. We will also prove, in contrast to the backward problems, that the solutions of problems (2) and (3) continuously depend on the right-hand side of the equation and on the function $\varphi$. Inequalities of coercivity type are obtained and it is shown that these inequalities differ depending on the considered type of fractional derivatives. The inverse problems of determining the right-hand side of the equation and function $\varphi$ in the boundary conditions are investigated.

The inverse problem of determining the source function $f$ with the final time observation has been well studied and much theoretical research has been published for classical partial differential equations. As a monograph, we should refer to Kabanikhin [6] and Prilepko, Orlovsky, and Vasin [16]. As for the fractional differential equations, one can construct theories parallel to [6,16], and the works are now made continuously. We give a brief overview of work on this inverse problem at the beginning of Section 4 .

The inverse problem of defining the function $\varphi$ arises from real-life processes. For example, when the initial temperature and final temperature for the heat equation are not indicated immediately, and it is not required to find, but information about the difference between the initial and final temperatures is sought. To the best of our knowledge, such an inverse problem was discussed only in the paper [17]. The authors considered this problem for the subdiffusion equation including the Caputo fractional derivative, the elliptical part of which is a two-variable differential expression with constant coefficients.

The remainder of this paper is composed of four sections and the Conclusion. In the next section, we introduce the Hilbert space associated with the degree of operator A and recall some properties of the Mittag-Leffler functions. Section 3 is devoted to the study of the nonlocal problem (2). Here, we first investigate problem (2) for the homogeneous equation, and then move on to the main problem. In Section 4, we study the inverse problem of determining the right-hand side of Equation (2). In this case, we assume that the unknown function $f$ does not depend on $t$. The next section is devoted to the study of the inverse problem for the determination of the boundary function $\varphi$. Since problems (2) and (3) are studied in a similar way, in Section 6 we present only the main points of the proof of the theorem on the existence and uniqueness of the solution to problem (3). Inverse problems for Equation (3) are considered in the same way as inverse problems for Equation (2). Therefore, we omit these details.

\section{Preliminaries}

In this section, we introduce the Hilbert space of "smooth" functions related to the degree of operator $A$ and recall some properties of the Mittag-Leffler functions, which we will use in what follows. 
Let $\tau$ be an arbitrary real number. We introduce the power of operator $A$, acting in $H$ according to the rule

$$
A^{\tau} h=\sum_{k=1}^{\infty} \lambda_{k}^{\tau} h_{k} v_{k}
$$

where $h_{k}$ are the Fourier coefficients of a function $h \in H: h_{k}=\left(h, v_{k}\right)$. Obviously, the domain of this operator has the form

$$
D\left(A^{\tau}\right)=\left\{h \in H: \sum_{k=1}^{\infty} \lambda_{k}^{2 \tau}\left|h_{k}\right|^{2}<\infty\right\}
$$

For elements of $D\left(A^{\tau}\right)$ we introduce the norm

$$
\|h\|_{\tau}^{2}=\sum_{k=1}^{\infty} \lambda_{k}^{2 \tau}\left|h_{k}\right|^{2}=\left\|A^{\tau} h\right\|^{2},
$$

and together with this norm $D\left(A^{\tau}\right)$ turns into a Hilbert space.

For $0<\rho<1$ and an arbitrary complex number $\mu$, by $E_{\rho, \mu}(z)$ we denote the MittagLeffler function with two parameters (see, e.g., [18], p. 12):

$$
E_{\rho, \mu}(z)=\sum_{n=0}^{\infty} \frac{z^{n}}{\Gamma(\rho n+\mu)}
$$

If the parameter $\mu=1$, then we have the classical Mittag-Leffler function: $E_{\rho}(z)=E_{\rho, 1}(z)$.

In what follows we need the asymptotic estimate of the Mittag-Leffler function with a sufficiently large negative argument. The well known estimate has the form (see, e.g., [19], p. 136)

$$
\left|E_{\rho, \mu}(-t)\right| \leq \frac{C}{1+t}, \quad t>0,
$$

where $\mu$ is an arbitrary complex number. This estimate essentially follows from the following asymptotic estimate (see, e.g., [19], p. 134):

$$
E_{\rho, \mu}(-t)=\frac{t^{-1}}{\Gamma(\mu-\rho)}+O\left(t^{-2}\right)
$$

For the Mittag-Leffler function with two parameters $E_{\rho, \rho}(-t)$ one can obtain a better estimate than (7). Indeed, using the asymptotic estimate (see, e.g., [19], p. 134)

$$
E_{\rho, \rho}(-t)=-\frac{t^{-2}}{\Gamma(-\rho)}+O\left(t^{-3}\right)
$$

and the fact that $E_{\rho, \rho}(t)$ is real analytic, we can obtain the following inequality [5]

$$
\left|E_{\rho, \rho}(-t)\right| \leq \frac{C}{1+t^{2}}, \quad t>0
$$

We will also use a coarser estimate with positive number $\lambda$ and $0<\varepsilon<1$ :

$$
\left|t^{\rho-1} E_{\rho, \rho}\left(-\lambda t^{\rho}\right)\right| \leq \frac{C t^{\rho-1}}{1+\left(\lambda t^{\rho}\right)^{2}} \leq C \lambda^{\varepsilon-1} t^{\varepsilon \rho-1}, \quad t>0
$$

which is easy to verify. Indeed, let $t^{\rho} \lambda<1$, then $t<\lambda^{-1 / \rho}$ and

$$
t^{\rho-1}=t^{\rho-\varepsilon \rho} t^{\varepsilon \rho-1}<\lambda^{\varepsilon-1} t^{\varepsilon \rho-1} .
$$


If $t^{\rho} \lambda \geq 1$, then $\lambda^{-1} \leq t^{\rho}$ and

$$
\lambda^{-2} t^{-\rho-1}=\lambda^{-1+\varepsilon} \lambda^{-1-\varepsilon} t^{-\rho-1} \leq \lambda^{\varepsilon-1} t^{\varepsilon \rho-1} .
$$

Proposition 1. Let $0<\rho<1$. Then

$$
E_{\rho}(x)>0, \quad \frac{d}{d x} E_{\rho}(x)>0, \quad x \in \mathbb{R} .
$$

Proof. For $x \geq 0$ this is obvious; estimates (12) follow from definition (6).

For $x<0$ we use the integral representation (see, e.g., [20], p. 54)

$$
E_{\rho}(x)=\frac{\sin \rho \pi}{\pi} \int_{0}^{\infty} \frac{e^{-t|x|^{1 / \rho}}}{1+2 t^{\rho} \cos \rho \pi+t^{2 \rho}} t^{\rho} d t>0 .
$$

Then

$$
\frac{d}{d x} E_{\rho}(x)=|x|^{(1-\rho) / \rho} \cdot \frac{\sin \rho \pi}{\rho \pi} \int_{0}^{\infty} \frac{e^{-t|x|^{1 / \rho}}}{1+2 t^{\rho} \cos \rho \pi+t^{2 \rho}} t^{\rho+1} d t>0 .
$$

Using Proposition 1 , by virtue of estimates (12) and equality $E_{\rho}(0)=1$, we arrive at (see [20], p. 47).

Proposition 2. The Mittag-Leffler function of the negative argument $E_{\rho}(-x)$ is monotonically decreasing function for all $0<\rho<1$ and

$$
0<E_{\rho}(-x)<1
$$

Proposition 3. Let $\rho>0$ and $\lambda>0$. Then for all positive $t>0$ one has [3]

$$
\int_{0}^{t} \eta^{\rho-1} E_{\rho, \rho}\left(-\lambda \eta^{\rho}\right) d \eta=\frac{1}{\lambda}\left(1-E_{\rho}\left(-\lambda t^{\rho}\right)\right)
$$

Proof. First, we calculate the derivative of the Mittag-Leffler function

$$
\frac{d}{d t} E_{\rho}\left(-\lambda t^{\rho}\right)=-\rho \lambda t^{\rho-1} \sum_{n=1}^{\infty} \frac{n\left(-\lambda t^{\rho}\right)^{n-1}}{\Gamma(\rho n+1)}=-\rho \lambda t^{\rho-1} \sum_{k=0}^{\infty} \frac{(k+1)\left(-\lambda t^{\rho}\right)^{k}}{\Gamma(\rho(k+1)+1)}=
$$

(since $\Gamma(x+1)=x \Gamma(x))$

$$
=-\lambda t^{\rho-1} \sum_{k=0}^{\infty} \frac{\left(-\lambda t^{\rho}\right)^{k}}{\Gamma(\rho k+\rho)}=-\lambda t^{\rho-1} E_{\rho, \rho}\left(-\lambda t^{\rho}\right)
$$

Note that here the series is termwise differentiable in $\mathbb{R}$.

Now, by virtue of the equality

$$
\int_{0}^{t} \eta^{\rho-1} E_{\rho, \rho}\left(-\lambda \eta^{\rho}\right) d \eta=-\frac{1}{\lambda} \int_{0}^{t} \frac{d}{d \eta} E_{\rho}\left(-\lambda \eta^{\rho}\right) d \eta,
$$

we obtain the required result. 
Proposition 4. Let $0<\rho<1$ and $\lambda>0$. Then

$$
\frac{d}{d t}\left[t^{\rho} E_{\rho, \rho+1}\left(-\lambda t^{\rho}\right)\right]>0, t>0,
$$

i.e., $t^{\rho} E_{\rho, \rho+1}\left(-\lambda t^{\rho}\right)$ strictly increases as a function of $t>0$.

Proof. Using (6) and term-by-term integration we arrive at (see [20], formula (4.4.4))

$$
\int_{0}^{t} \eta^{\rho-1} E_{\rho, \rho}\left(-\lambda \eta^{\rho}\right) d \eta=t^{\rho} E_{\rho, \rho+1}\left(-\lambda t^{\rho}\right)
$$

or by Proposition 3,

$$
t^{\rho} E_{\rho, \rho+1}\left(-\lambda t^{\rho}\right)=\frac{1}{\lambda}\left(1-E_{\rho}\left(-\lambda t^{\rho}\right)\right) .
$$

It remains to apply Proposition 1.

Proposition 5. Let $0<\rho<1$ and $\lambda>0$. Then for all positive t one has

$$
\partial_{t}^{\rho-1}\left(t^{\rho-1} E_{\rho, \rho}\left(-\lambda t^{\rho}\right)\right)=E_{\rho}\left(-\lambda t^{\rho}\right) .
$$

Proof. By the definition of fractional integration (1) we have

$$
\begin{gathered}
\partial_{t}^{\rho-1}\left(t^{\rho-1} E_{\rho, \rho}\left(-\lambda t^{\rho}\right)\right)=\frac{1}{\Gamma(1-\rho)} \int_{0}^{t} \frac{\xi^{\rho-1} E_{\rho, \rho}\left(-\lambda \xi^{\rho}\right)}{(t-\xi)^{\rho}} d \xi= \\
=\frac{1}{\Gamma(1-\rho)} \sum_{j=0}^{\infty} \frac{(-\lambda)^{j}}{\Gamma(\rho j+\rho)} \int_{0}^{t} \frac{\xi^{\rho-1+\rho j}}{(t-\xi)^{\rho}} d \xi= \\
=\frac{1}{\Gamma(1-\rho)} \sum_{j=0}^{\infty} \frac{(-\lambda)^{j}}{\Gamma(\rho j+\rho)} t^{\rho j} \int_{0}^{1} s^{\rho-1+\rho j}(1-s)^{-\rho} d s .
\end{gathered}
$$

On the other hand, using the properties of Euler's beta function $B(a, b)$, we obtain

$$
B(\rho+\rho j, 1-\rho)=\int_{0}^{1} s^{\rho-1+\rho j}(1-s)^{-\rho} d s=\frac{\Gamma(\rho+\rho j) \Gamma(1-\rho)}{\Gamma(\rho j+1)} .
$$

By virtue of the definition of the Mittag-Leffler function $E_{\rho}(z)$ this implies the statement of the proposition.

\section{Well-Posedness of Problem (2)}

To solve problem (2), we divide it into two auxiliary problems:

$$
\left\{\begin{array}{l}
D_{t}^{\rho} \omega(t)+A \omega(t)=f(t), \quad 0<t \leq T \\
\omega(0)=0
\end{array}\right.
$$

and

$$
\left\{\begin{array}{l}
D_{t}^{\rho} w(t)+A w(t)=0, \quad 0<t \leq T \\
w(\xi)=\alpha w(0)+\psi, \quad 0<\xi \leq T
\end{array}\right.
$$

where $\psi \in H$ is a given function. 
Problem (16) is a special case of problem (2), and the solution to problem (15) is defined similarly to Definition 1.

If $\psi=\varphi-\omega(\xi)$ and $\omega(t)$ and $w(t)$ are the corresponding solutions, then it is easy to verify that function $u(t)=\omega(t)+w(t)$ is a solution to problem (2). Therefore, it is sufficient to solve the auxiliary problems.

For problem (15) we have the following statement.

Theorem 1. Let $f(t) \in C\left([0, T] ; D\left(A^{\varepsilon}\right)\right)$ for some $\varepsilon \in(0,1)$. Then problem (15) has a unique solution and this solution has the representation

$$
\omega(t)=\sum_{k=1}^{\infty}\left[\int_{0}^{t} \eta^{\rho-1} E_{\rho, \rho}\left(-\lambda_{k} \eta^{\rho}\right) f_{k}(t-\eta) d \eta\right] v_{k} .
$$

Moreover, there is a constant $C_{\varepsilon}>0$ such that the following coercive type inequality holds:

$$
\left\|D_{t}^{\rho} \omega(t)\right\|^{2}+\|\omega(t)\|_{1}^{2} \leq C_{\varepsilon} \max _{t \in[0, T]}\|f\|_{\varepsilon}^{2}, \quad 0<t \leq T
$$

Proof of Theorem 1. It is not hard to verify that the series (17) is a formal solution to problem (15) (see, e.g., [20], p. 173, [21]). In order to prove that function (17) is actually a solution to the problem, it remains to substantiate this formal statement, i.e., to show that the operators $A$ and $D_{t}^{\rho}$ can be applied term-by-term to series (17).

Let $S_{j}(t)$ be the partial sum of series (17). Then

$$
A S_{j}(t)=\sum_{k=1}^{j}\left[\int_{0}^{t} \eta^{\rho-1} E_{\rho, \rho}\left(-\lambda_{k} \eta^{\rho}\right) f_{k}(t-\eta) d \eta\right] \lambda_{k} v_{k}
$$

Due to the Parseval equality we may write

$$
\left\|A S_{j}(t)\right\|^{2}=\sum_{k=1}^{j} \lambda_{k}^{2}\left|\int_{0}^{t} \eta^{\rho-1} E_{\rho, \rho}\left(-\lambda_{k} \eta^{\rho}\right) f_{k}(t-\eta) d \eta\right|^{2} .
$$

Then, by inequality (11) for $0<\varepsilon<1$ one has

$$
\left\|A S_{j}(t)\right\|^{2} \leq C \sum_{k=1}^{j}\left[\int_{0}^{t} \eta^{\varepsilon \rho-1} \lambda_{k}^{\varepsilon}\left|f_{k}(t-\eta)\right| d \eta\right]^{2}
$$

or, by virtue of the generalized Minkowski inequality,

$$
\left\|A S_{j}(t)\right\|^{2} \leq C\left[\int_{0}^{t} \eta^{\varepsilon \rho-1}\left(\sum_{k=1}^{j}\left|\lambda_{k}^{\varepsilon} f_{k}(t-\eta)\right|^{2}\right)^{\frac{1}{2}} d \eta\right]^{2} \leq C_{\varepsilon} \max _{t \in[0, T]}\|f(t)\|_{\varepsilon}^{2} .
$$

Hence, we obtain $A \omega(t) \in C([0, T] ; H)$ and in particular $\omega(t) \in C([0, T] ; H)$.

Furthermore, from Equation (2) one has $D_{t}^{\rho} S_{j}(t)=-A S_{j}(t)+\sum_{k=1}^{j} f_{k}(t) v_{k}, t>0$. Therefore, from the above reasoning, we have $D_{t}^{\rho} \omega(t) \in C((0, T] ; H)$ and

$$
\left\|D_{t}^{\rho} S_{j}(t)\right\|^{2} \leq C_{\varepsilon} \max _{t \in[0, T]}\|f(t)\|_{\varepsilon}^{2}+\|f(t)\|^{2}, \quad t>0 .
$$

Thus, we have completed the rationale that (17) is a solution to problem (15). The last two inequalities imply estimate (18). 
The uniqueness of the solution can be proved by the standard technique based on completeness of the set of eigenfunctions $\left\{v_{k}\right\}$ in $H$ (see, e.g., [5]).

Theorem 1 is completely proved.

If $f$ does not depend on $t$, then the statement of Theorem 1 is true for all $f \in H$.

Corollary 1. Let $f \in H$. Then problem (15) has a unique solution and this solution has the representation

$$
\omega(t)=\sum_{k=1}^{\infty} f_{k} t^{\rho} E_{\rho, \rho+1}\left(-\lambda_{k} t^{\rho}\right) v_{k} .
$$

Moreover, there is a positive constant $C$ such that the following coercive type inequality holds:

$$
\left\|D_{t}^{\rho} \omega(t)\right\|^{2}+\|\omega(t)\|_{1}^{2} \leq C\|f\|^{2}, \quad 0<t \leq T .
$$

Proof. Since $f$ does not depend on $t$, then we have the following form for the Fourier coefficients of $\omega$ (see (17))

$$
\omega_{k}(t)=f_{k} \int_{0}^{t} \eta^{\rho-1} E_{\rho, \rho}\left(-\lambda_{k} \eta^{\rho}\right) d \eta .
$$

Application of Formula (14) to the integral shows that the formal solution to problem (15) has the form (20).

Let $S_{j}(t)$ be the partial sum of series (20). Then by virtue of estimate (7), we obtain

$$
\left\|A S_{j}(t)\right\|^{2} \leq C \sum_{k=1}^{j}\left|\frac{\lambda_{k} t^{\rho} f_{k}}{1+\lambda_{k} t^{\rho}}\right|^{2} \leq C\|f\|^{2} .
$$

Now, using this estimate and repeating the arguments similar to the proof of Theorem 1 , it is easy to check that (20) is indeed a solution to problem (15) and estimate (21) holds true.

We now turn to problem (16). In accordance with the Fourier method, we will look for a solution to problem (16) in the form of a series:

$$
w(t)=\sum_{k=1}^{\infty} T_{k}(t) v_{k}
$$

where $T_{k}(t), k \geq 1$, are solutions of the nonlocal problems:

$$
\left\{\begin{array}{l}
D_{t}^{\rho} T_{k}(t)+\lambda_{k} T_{k}(t)=0, \quad 0<t \leq T ; \\
T_{k}(\xi)=\alpha T_{k}(0)+\psi_{k},
\end{array}\right.
$$

where $\psi_{k}$ are the Fourier coefficients of function $\psi \in H$.

Let us denote $T_{k}(0)=b_{k}$. Then the unique solution to the differential Equation (23) with this initial condition has the form $T_{k}(t)=b_{k} E_{\rho}\left(-\lambda_{k} t^{\rho}\right)$ (see, e.g., [20], p. 174). From the nonlocal conditions of (23) we obtain the following equation to find the unknown numbers $b_{k}$ :

$$
b_{k} E_{\rho}\left(-\lambda_{k} \xi^{\rho}\right)=\alpha b_{k}+\psi_{k} .
$$

By virtue of property (13) of the Mittag-Leffler function, $E_{\rho}\left(-\lambda_{k} \xi^{\rho}\right) \neq \alpha$ for all $\alpha \geq 1$ and $\alpha<0$ (note, $\xi>0$ and $\lambda_{k}>0$ ). Therefore, from (24) we have

$$
b_{k}=\frac{\psi_{k}}{E_{\rho}\left(-\lambda_{k} \xi^{\rho}\right)-\alpha}, \quad\left|b_{k}\right| \leq C_{\alpha}\left|\psi_{k}\right|, \quad k \geq 1, \quad \text { and } \quad \alpha \geq 1 \quad \text { or } \quad \alpha<0,
$$


here and below, by $C_{\beta}$ we will denote a constant depending on $\beta$, not necessarily the same one.

If $\alpha=0$, then $E_{\rho}\left(-\lambda_{k} \xi^{\rho}\right) \neq 0$, but the Mittag-Leffler function can asymptotically tend towards zero (see (8)). Therefore, in this case one has:

$$
b_{k}=\frac{\psi_{k}}{E_{\rho}\left(-\lambda_{k} \xi^{\rho}\right)}, \quad\left|b_{k}\right| \leq C_{\rho} \lambda_{k} \xi^{\rho}\left|\psi_{k}\right| .
$$

This case, as noted above (see (4)), has been studied in detail in [2-4].

Let $0<\alpha<1$. Then according to Proposition 2, there is a unique $\lambda_{0}>0$ such that $E_{\rho}\left(-\lambda_{0} \xi^{\rho}\right)=\alpha$. If $\lambda_{k} \neq \lambda_{0}$ for all $k \geq 1$, then the estimate in (25) holds with some constant $C_{\alpha}>0$.

Thus, if $\alpha \notin(0,1)$ or $\alpha \in(0,1)$, but $\lambda_{k} \neq \lambda_{0}$ for all $k \geq 1$, then the formal solution of problem (16) has the form

$$
w(t)=\sum_{k=1}^{\infty} \frac{\psi_{k}}{E_{\rho}\left(-\lambda_{k} \xi^{\rho}\right)-\alpha} E_{\rho}\left(-\lambda_{k} t^{\rho}\right) v_{k} .
$$

Finally, let $0<\alpha<1$ and $\lambda_{k}=\lambda_{0}$ for $k=k_{0}, k_{0}+1, \ldots, k_{0}+p_{0}-1$, where $p_{0}$ is the multiplicity of the eigenvalue $\lambda_{k_{0}}$. Then the nonlocal problem (23) has a solution if the boundary function $\psi(x)$ satisfies the following orthogonality conditions

$$
\psi_{k}=\left(\psi, v_{k}\right)=0, k \in K_{0} ; K_{0}=\left\{k_{0}, k_{0}+1, \ldots, k_{0}+p_{0}-1\right\},
$$

and for these $k \in K_{0}$ arbitrary numbers $b_{k}$ are solutions of Equation (24). For all other $k$ we have

$$
b_{k}=\frac{\psi_{k}}{E_{\rho}\left(-\lambda_{k} \xi^{\rho}\right)-\alpha}, \quad\left|b_{k}\right| \leq C_{\alpha}\left|\psi_{k}\right|, \quad k \notin K_{0} .
$$

Thus, the formal solution of problem (16) in this case has the form

$$
w(t)=\sum_{k \notin K_{0}} \frac{\psi_{k}}{E_{\rho}\left(-\lambda_{k} \xi^{\rho}\right)-\alpha} E_{\rho}\left(-\lambda_{k} t^{\rho}\right) v_{k}+\sum_{k \in K_{0}} b_{k} E_{\rho}\left(-\lambda_{k} t^{\rho}\right) v_{k} .
$$

Throughout what follows we will assume that whenever $0<\alpha<1$ and $\lambda_{k}=\lambda_{0}$, then orthogonality condition (27) is satisfied.

Let us show that the operators $A$ and $D_{t}^{\rho}$ can be applied term-by-term to series (26); for series (29) this question is considered in a completely similar way.

Let $S_{j}(t)$ be the partial sum of series (26). Then

$$
A S_{j}(t)=\sum_{k=1}^{j} \lambda_{k} \frac{\psi_{k}}{E_{\rho}\left(-\lambda_{k} \xi^{\rho}\right)-\alpha} E_{\rho}\left(-\lambda_{k} t^{\rho}\right) v_{k}
$$

Due to the Parseval equality, we may write

$$
\left\|A S_{j}(t)\right\|^{2} \leq \sum_{k=1}^{j} \lambda_{k}^{2}\left|\frac{\psi_{k}}{E_{\rho}\left(-\lambda_{k} \xi^{\rho}\right)-\alpha} E_{\rho}\left(-\lambda_{k} t^{\rho}\right)\right|^{2} .
$$

Using estimates (7), (25) and (28) we obtain

$$
\left\|A S_{j}(t)\right\|^{2} \leq C_{\alpha} \sum_{k=1}^{j} \lambda_{k}^{2}\left|\frac{\psi_{k}}{1+\lambda_{k} t^{\rho}}\right|^{2} \leq C_{\alpha} t^{-2 \rho} \sum_{k=1}^{j}\left|\psi_{k}\right|^{2} .
$$


Therefore if $\psi \in H$, then $A u(t) \in C((0, T] ; H)$. From Equation (16) one has $D_{t}^{\rho} u(t)=$ $-A u(t), t>0$, and the above estimates imply

$$
\left\|D_{t}^{\rho} w(t)\right\|^{2} \leq C_{\alpha} t^{-2 \rho} \sum_{k=1}^{j}\left|\psi_{k}\right|^{2},
$$

which means $D_{t}^{\rho} w(t) \in C((0, T] ; H)$.

For $S_{j}(t)$, taking into account estimate $(7)$, we obtain

$$
\left\|S_{j}(t)\right\|^{2} \leq C_{\alpha} \sum_{k=1}^{j}\left|\psi_{k}\right|^{2} .
$$

Hence $w(t) \in C([0, T] ; H)$, which was required by the definition of the solution to problem (16).

Let us investigate the uniqueness of the solution to problem (16). Suppose we have two solutions: $w_{1}(t), w_{2}(t)$ and set $w(t)=w_{1}(t)-w_{2}(t)$. Then, we have

$$
\left\{\begin{array}{l}
D_{t}^{\rho} w(t)+A w(t)=0, \quad 0<t \leq T \\
w(\xi)=\alpha w(0), \quad 0<\xi \leq T .
\end{array}\right.
$$

Let $w_{k}(t)=\left(w(t), v_{k}\right)$. Since the operator $A$ is self-adjoint, one has

$$
D_{t}^{\rho} w_{k}(t)=\left(D_{t}^{\rho} w(t), v_{k}\right)=-\left(A w(t), v_{k}\right)=-\left(w(t), A v_{k}\right)=-\lambda_{k} w_{k}(t)
$$

and the nonlocal condition implies

$$
w_{k}(\xi)=\alpha w_{k}(0)
$$

Let us denote $w_{k}(0)=b_{k}$. Then the unique solution to the differential Equation (35) with this initial condition has the form $w_{k}(t)=b_{k} E_{\rho}\left(-\lambda_{k} t^{\rho}\right)$ (see, e.g., [20], p. 174). From the nonlocal conditions of (36) we obtain the following equation to find the unknown numbers $b_{k}$ :

$$
b_{k} E_{\rho}\left(-\lambda_{k} \xi^{\rho}\right)=\alpha b_{k} .
$$

Let first $\alpha \notin(0,1)$ or $\alpha \in(0,1)$, but $\lambda_{k} \neq \lambda_{0}$ for all $k \geq 1$. Then $E_{\rho}\left(-\lambda_{k} \xi^{\rho}\right) \neq \alpha$ for all $k$. Consequently, in this case all $b_{k}$ are equal to zero (therefore $w_{k}(t)=0$ ), and by virtue of completeness of the set of eigenfunctions $\left\{v_{k}\right\}$, we conclude that $w(t) \equiv 0$. Thus, problem (16) in this case has a unique solution.

Now suppose that $\alpha \in(0,1)$ and $\lambda_{k}=\lambda_{0}, k \in K_{0}$. Then $E_{\rho}\left(-\lambda_{k} \xi^{\rho}\right)=\alpha, k \in K_{0}$ and therefore Equation (37) has the following solution: $b_{k}=0$ if $k \notin K_{0}$ and $b_{k}$ is an arbitrary number for $k \in K_{0}$. Thus, in this case, there is no uniqueness of the solution to problem (16).

Thus, we obtain the following statement:

Theorem 2. Let $\psi \in H$.

If $\alpha \notin(0,1)$ or $\alpha \in(0,1)$, but $\lambda_{k} \neq \lambda_{0}$ for all $k \geq 1$, then problem (16) has a unique solution and this solution has the form (26).

If $\alpha \in(0,1)$ and $\lambda_{k}=\lambda_{0}, k \in K_{0}$, then we assume that the orthogonality conditions (27) are satisfied. The solution of problem (16) has the form (29) with arbitrary coefficients $b_{k}, k \in K_{0}$.

Moreover, there is a constant $C_{\alpha}>0$ such that the following coercive type inequality holds:

$$
\left\|D_{t}^{\rho} w(t)\right\|^{2}+\|w(t)\|_{1}^{2} \leq C_{\alpha} t^{-2 \rho}\|\psi\|^{2}, \quad 0<t \leq T .
$$

Note that the proof of the coercive type inequality (38) follows from the estimates (31) and (32). 
Now let us move on to solving the main problem (2). Let $\varphi \in H$ and $f(t) \in$ $C\left([0, T] ; D\left(A^{\varepsilon}\right)\right)$ for some $\varepsilon \in(0,1)$. As noted above, if we put $\psi=\varphi-\omega(\xi) \in H$ and $\omega(t)$ and $w(t)$ are the corresponding solutions of problems (15) and (16), then function $u(t)=\omega(t)+w(t)$ is a solution to problem (2). Therefore, if $\alpha \notin(0,1)$ or $\alpha \in(0,1)$, but $\lambda_{k} \neq \lambda_{0}$ for all $k \geq 1$, then

$$
u(t)=\sum_{k=1}^{\infty}\left[\frac{\varphi_{k}-\omega_{k}(\xi)}{E_{\rho}\left(-\lambda_{k} \xi^{\rho}\right)-\alpha} E_{\rho}\left(-\lambda_{k} t^{\rho}\right)+\omega_{k}(t)\right] v_{k}
$$

where

$$
\omega_{k}(t)=\int_{0}^{t} \eta^{\rho-1} E_{\rho, \rho}\left(-\lambda_{k} \eta^{\rho}\right) f_{k}(t-\eta) d \eta
$$

The uniqueness of the function $u(t)$ follows from the uniqueness of the solutions $\omega(t)$ and $w(t)$.

If $\alpha \in(0,1)$ and $\lambda_{k}=\lambda_{0}, k \in K_{0}$, then

$$
u(t)=\sum_{k \notin K_{0}}\left[\frac{\varphi_{k}-\omega_{k}(\xi)}{E_{\rho}\left(-\lambda_{k} \xi^{\rho}\right)-\alpha} E_{\rho}\left(-\lambda_{k} t^{\rho}\right)+\omega_{k}(t)\right] v_{k}+\sum_{k \in K_{0}} b_{k} E_{\rho}\left(-\lambda_{k} t^{\rho}\right) v_{k} .
$$

The corresponding orthogonality conditions have the form

$$
\left(\varphi, v_{k}\right)=\left(\omega(\xi), v_{k}\right), k \in K_{0} ; K_{0}=\left\{k_{0}, k_{0}+1, \ldots, k_{0}+p_{0}-1\right\} .
$$

In particular, if

$$
\left(\varphi, v_{k}\right)=0,\left(f(t), v_{k}\right)=0, \text { for all } t>0, k \in K_{0} ; K_{0}=\left\{k_{0}, k_{0}+1, \ldots, k_{0}+p_{0}-1\right\},
$$

then the orthogonality conditions (41) are satisfied.

Thus we have proved the main result of this section:

Theorem 3. Let $\varphi \in H$ and $f(t) \in C\left([0, T] ; D\left(A^{\varepsilon}\right)\right)$ for some $\varepsilon \in(0,1)$.

If $\alpha \notin(0,1)$ or $\alpha \in(0,1)$, but $\lambda_{k} \neq \lambda_{0}$ for all $k \geq 1$, then problem (2) has a unique solution and this solution has the form (39).

If $\alpha \in(0,1)$ and $\lambda_{k}=\lambda_{0}, k \in K_{0}$, then we assume that the orthogonality conditions (42) are satisfied. The solution of problem (16) has the form (40) with arbitrary coefficients $b_{k}, k \in K_{0}$.

Moreover, there are constants $C_{\alpha}>0$ and $C_{\varepsilon}>0$ such that the following coercive type inequality holds:

$$
\left\|D_{t}^{\rho} u(t)\right\|^{2}+\|u(t)\|_{1}^{2} \leq C_{\alpha} t^{-2 \rho}\|\varphi\|^{2}+C_{\varepsilon} \max _{t \in[0, T]}\|f\|_{\varepsilon}^{2}, \quad 0<t \leq T .
$$

The results of Theorems 2 and 3 are based on the assumption of orthogonality conditions (27) and (42) correspondingly. The question naturally arise, to what extent do these assumtions limit? In order to answer this question, consider the following example.

Let $\Omega \subset R^{N}$ be a bounded domain with sufficiently smooth boundary $\partial \Omega$ and denote by $A_{0}$ the operator in $L_{2}(\Omega)$ with domain of definition $D\left(A_{0}\right)=\left\{f \in C^{2}(\Omega) \cap C(\bar{\Omega})\right.$ : $f(x)=0, x \in \partial \Omega\}$ and acting as $A_{0} f(x)=-\triangle f(x)$, where $\triangle$ is the Laplace operator. Then (see, e.g., [22]) $A_{0}$ has a complete in $L_{2}(\Omega)$ system of orthonormal eigenfunctions $\left\{v_{k}(x)\right\}$ and a countable set of nonnegative eigenvalues $\lambda_{k}(\rightarrow+\infty)$, and $\lambda_{1}=\lambda_{1}(\Omega)>0$.

Let $A$ stand for the operator, acting as $A f(x)=\sum \lambda_{k} f_{k} v_{k}(x)$ with the domain of definition $D(A)=\left\{f \in L_{2}(\Omega): \sum \lambda_{k}^{2} f_{k}^{2}<\infty\right\}$. Then it is not hard to verify, that $A$ 
is a positive self-adjoint extension in $L_{2}(\Omega)$ of operator $A_{0}$. Therefore, one can apply Theorems 2 and 3 to operator $A$ and consequently to the problem:

$$
\left\{\begin{array}{l}
D_{t}^{\rho} w(x, t)-\Delta w(x, t)=0, \quad x \in \Omega, \quad 0<t \leq T \\
w(x, \xi)=\alpha w(x, 0)+\psi(x), \quad x \in \Omega, \quad 0<\xi \leq T \\
w(x, t)=0, \quad x \in \partial \Omega, \quad 0<t \leq T,
\end{array}\right.
$$

Suppose $\alpha \in(0,1)$ and $\xi \in(0, T]$ are such that

$$
E_{\rho}\left(-\lambda_{1} \xi^{\rho}\right)=\alpha
$$

Then problem (44) has the unique solution for any function $\psi \in L_{2}(\Omega)$, which satisfies the orthogonality condition

$$
\int_{\Omega} \psi(x) v_{1}(x) d x=0,
$$

or in other words, for any function

$$
\psi(x)=\sum_{k=2}^{\infty} \psi_{k} v_{k}(x),
$$

with

$$
\sum_{k=2}^{\infty}\left|\psi_{k}\right|^{2}<\infty .
$$

\section{Inverse Problem of Determining the Heat Source Density}

The inverse problems of determining the right-hand side (the heat source density) of various subdiffusion equations have been considered by a number of authors (see, e.g., survey papers [23] and the bibliography therein). However, there is no general closed theory for the abstract case of the source function $F(x, t)$. Known results deal with the separated source term $F(x, t)=h(t) f(x)$. The appropriate choice of the overdetermination depends on the choice whether the unknown is $h(t)$ or $f(x)$.

The most difficult to study is the case when the function $h(t)$ is unknown (see the survey work [3,23] for the case of subdiffusion equations, and, for example, monographs $[6,16]$ and [24-26] for the classical heat equation). In inverse problems of this type, the condition $u\left(x_{0}, t\right)=u_{0}(t)$ is taken as an additional one, and the operator $A$ does not depend on $t$. In all the above-mentioned works on the subdiffusion equations, only the uniqueness of the solution to the inverse problem was proved.

Uniqueness questions in the inverse problem of finding the function $f(x)$ in fractional diffusion equations

$$
D_{t}^{\rho} u+A u=h(t) f(x),
$$

has been studied in, e.g., $[27,28]$. Paper [27] shows the uniqueness result for $f(x)$ if $h(t)>0$ in a case of a self-adjoint operator $A$ with time-independent coefficients. Article [28], Example 3.1, shows the non-uniqueness result if $\mathrm{h}(\mathrm{t})$ changes its sign. The time- dependent operator $A(t)$ has been considered in [28], Theorem 3.1, where uniqueness was established under some monotonic in time assumptions on $h(t)$ and $A(t)$. In paper [29], the author studies a general multi-dimensional case for a linear time-fractional partial differential equations with time-dependent coeffitients. The author uses separation of variable technique combined with the maximum principle to derive the uniqueness result assuming $h \in C[0, T]$ and $0 \not \equiv h \geq 0$. In this result, we do not need any monotonic premises on $h(t)$ and/or the coefficients of $A(t)$, which is the new aspect (and highlight) in this area of inverse problems.

Many authors have considered an Equation (45) in which $h(t) \equiv 1$ and $f(x)$ is unknown (see, e.g., [30-48]). Let us mention only some of these works. The case of subdif- 
fusion equations, the elliptic part of $A$ of which is an ordinary differential expression, is considered in [30-36]. The authors of the articles [37-41] studied subdiffusion equations in which the elliptic part of $A$ is either a Laplace operator or a second-order operator. The paper [42] studied the inverse problem for the subdiffusion Equation (2) with the Cauchy condition. In this article [42] and most other articles, including [37-40], the Caputo derivative is used as a fractional derivative. The recent articles $[43,44]$ are devoted to the inverse problem for the subdiffusion equation with Riemann-Liouville derivatives. In [31,41], the fractional derivative in the subdiffusion equation is a two-parameter generalized Hilfer fractional derivative; this type of fractional derivative contains a parameter belonging to the interval [0,1], and its extreme values correspond to the Caputo and Riemann-Liouville derivatives. Various models of applied problems leading to Hilfer fractional derivatives are investigated in [49]. Note also that the papers [31,37,40] contain a survey of papers dealing with inverse problems of determining the right-hand side of the subdiffusion equation.

In $[41,46,47]$, non-self-adjoint differential operators (with nonlocal boundary conditions) were taken as $\mathrm{A}$, and solutions to the inverse problem were found in the form of biortagonal series.

In their previous work [48], the authors of this article considered an inverse problem for simultaneously determining the order of the Riemann-Liouville fractional derivative and the source function in the subdiffusion equations. Using the classical Fourier method, the authors proved the uniqueness and existence of a solution to this inverse problem.

It should be noted that in all of the listed works, the Cauchy conditions in time are considered (an exception is work [45], where the integral condition is set with respect to the variable $t$ ). In the present paper, for the best of our knowledge, an inverse problem for subdiffusion equation with a nonlocal condition in time (see (46)) is considered for the first time.

The papers $[50,51]$ deal with the inverse problem of determining the order of the fractional derivative in the subdiffusion equation and in the wave equation, respectively.

Let us consider the inverse problem

$$
\left\{\begin{array}{l}
D_{t}^{\rho} u(t)+A u(t)=f, \quad 0<t \leq T \\
u(\xi)=\alpha u(0)+\varphi, \quad 0<\xi \leq T
\end{array}\right.
$$

with the additional condition

$$
u(\tau)=\Psi, \quad 0<\tau \leq T, \quad \tau \neq \xi,
$$

in which the unknown element $f \in H$, characterizing the action of heat sources, does not depend on $t$ and $\Psi, \varphi \in H$ are given elements, $\alpha$ is an arbitrary given constant.

Note that if $\tau=\xi$, then the nonlocal condition in (46) coincides with the Cauchy condition $u(0)=\varphi_{1}$ (see (4)). In this case, this inverse problem was studied in [42].

Definition 2. A pair $\{u(t), f\}$ of functions $u(t) \in C([0, T] ; H)$ and $f \in H$ with the properties $D_{t}^{\rho} u(t), A u(t) \in C((0, T] ; H)$ and satisfying conditions (46), (47) is called the solution of the inverse problem (46), (47).

In what follows we shall deal only with the case $\alpha \geq 1$, since in this case the uniqueness of the solution is relatively easy to prove.

Theorem 4. Let $\varphi, \Psi \in D(A)$ and $\alpha \geq 1$. Then the inverse problem (46), (47) has a unique solution $\{u(t), f\}$ and this solution has the following form

$$
f=\sum_{k=1}^{\infty}\left[\frac{\alpha-E_{\rho}\left(-\lambda_{k} \xi^{\rho}\right)}{E_{\rho}\left(-\lambda_{k} \tau^{\rho}\right) \xi^{\rho} E_{\rho, \rho+1}\left(-\lambda_{k} \xi^{\rho}\right)+\tau^{\rho} E_{\rho, \rho+1}\left(-\lambda_{k} \tau^{\rho}\right)\left[\alpha-E_{\rho}\left(-\lambda_{k} \xi^{\rho}\right)\right]} \Psi_{k}+\right.
$$




$$
\begin{gathered}
\left.+\frac{E_{\rho}\left(-\lambda_{k} \tau^{\rho}\right)}{E_{\rho}\left(-\lambda_{k} \tau^{\rho}\right) \xi^{\rho} E_{\rho, \rho+1}\left(-\lambda_{k} \xi^{\rho}\right)+\tau^{\rho} E_{\rho, \rho+1}\left(-\lambda_{k} \tau^{\rho}\right)\left[\alpha-E_{\rho}\left(-\lambda_{k} \xi^{\rho}\right)\right]} \varphi_{k}\right] v_{k}, \\
u(t)=\sum_{k=1}^{\infty}\left[\frac{E_{\rho}\left(-\lambda_{k} t^{\rho}\right)}{E_{\rho}\left(-\lambda_{k} \xi^{\rho}\right)-\alpha}\left[\varphi_{k}-f_{k} \xi^{\rho} E_{\rho, \rho+1}\left(-\lambda_{k} \xi^{\rho}\right)\right]+f_{k} t^{\rho} E_{\rho, \rho+1}\left(-\lambda_{k} t^{\rho}\right)\right] v_{k} .
\end{gathered}
$$

Proof of Theorem 4. Existence. If $f$ is known, then the unique solution of problem (46) has the form (39), and since $f$ does not depend on $t$, then, thanks to formulas (22) and (14), it is easy to verify that the formal solution of problem (46) has the form (49).

By virtue of additional condition (47) and completeness of the system $\left\{v_{k}\right\}$ we obtain:

$$
\frac{E_{\rho}\left(-\lambda_{k} \tau^{\rho}\right)}{E_{\rho}\left(-\lambda_{k} \xi^{\rho}\right)-\alpha}\left[\varphi_{k}-f_{k} \xi^{\rho} E_{\rho, \rho+1}\left(-\lambda_{k} \xi^{\rho}\right)\right]+f_{k} \tau^{\rho} E_{\rho, \rho+1}\left(-\lambda_{k} \tau^{\rho}\right)=\Psi_{k} .
$$

After simple calculations, we obtain

$$
\begin{gathered}
f_{k}=\frac{\alpha-E_{\rho}\left(-\lambda_{k} \xi^{\rho}\right)}{E_{\rho}\left(-\lambda_{k} \tau^{\rho}\right) \xi^{\rho} E_{\rho, \rho+1}\left(-\lambda_{k} \xi^{\rho}\right)+\tau^{\rho} E_{\rho, \rho+1}\left(-\lambda_{k} \tau^{\rho}\right)\left[\alpha-E_{\rho}\left(-\lambda_{k} \xi^{\rho}\right)\right]} \Psi_{k}+ \\
+\frac{E_{\rho}\left(-\lambda_{k} \tau^{\rho}\right)}{E_{\rho}\left(-\lambda_{k} \tau^{\rho}\right) \xi^{\rho} E_{\rho, \rho+1}\left(-\lambda_{k} \xi^{\rho}\right)+\tau^{\rho} E_{\rho, \rho+1}\left(-\lambda_{k} \tau^{\rho}\right)\left[\alpha-E_{\rho}\left(-\lambda_{k} \xi^{\rho}\right)\right]} \varphi_{k} \equiv f_{k, 1}+f_{k, 2} .
\end{gathered}
$$

With these Fourier coefficients we have the above formal series (48) for the unknown function $f: f=\sum_{k=1}^{\infty}\left(f_{k, 1}+f_{k, 2}\right) v_{k}$.

Let us show the convergence of series (48). If $F_{j}$ are the partial sums of series (48), then by virtue of the Parseval equality we may write

$$
\left\|F_{j}\right\|^{2}=\sum_{k=1}^{j}\left[f_{k, 1}+f_{k, 2}\right]^{2} \leq 2 \sum_{k=1}^{j} f_{k, 1}^{2}+2 \sum_{k=1}^{j} f_{k, 2}^{2} \equiv 2 I_{1, j}+2 I_{2, j} .
$$

Since $\xi>0$, then $E_{\rho}\left(-\lambda_{k} \tau^{\rho}\right) \xi^{\rho} E_{\rho, \rho+1}\left(-\lambda_{k} \xi^{\rho}\right)>0$. Therefore,

$$
I_{1, j} \leq \sum_{k=1}^{j}\left|\frac{\alpha-E_{\rho}\left(-\lambda_{k} \xi^{\rho}\right)}{\tau^{\rho} E_{\rho, \rho+1}\left(-\lambda_{k} \tau^{\rho}\right)\left[\alpha-E_{\rho}\left(-\lambda_{k} \xi^{\rho}\right)\right]}\right|^{2}\left|\Psi_{k}\right|^{2}=\sum_{k=1}^{j} \frac{\left|\Psi_{k}\right|^{2}}{\left|\tau^{\rho} E_{\rho, \rho+1}\left(-\lambda_{k} \tau^{\rho}\right)\right|^{2}} .
$$

Using the asymptotic estimate (see (8))

$$
E_{\rho, \rho+1}(-t)=t^{-1}+O\left(t^{-2}\right),
$$

we obtain

$$
I_{1, j} \leq \sum_{k=1}^{j} \frac{\lambda_{k}^{2}\left|\Psi_{k}\right|^{2}}{\left(1+O\left(\left(-\lambda_{k} \tau^{\rho}\right)^{-1}\right)\right)^{2}} \leq C \sum_{k=1}^{j} \lambda_{k}^{2}\left|\Psi_{k}\right|^{2} \leq C|| \Psi \|_{1}^{2} .
$$

Since $\tau>0$ and $\alpha \geq 1$, then $\tau^{\rho} E_{\rho, \rho+1}\left(-\lambda_{k} \tau^{\rho}\right)\left[\alpha-E_{\rho}\left(-\lambda_{k} \xi^{\rho}\right)\right]>0$. Therefore,

$$
I_{2, j} \leq \sum_{k=1}^{j}\left|\frac{E_{\rho}\left(-\lambda_{k} \tau^{\rho}\right)}{E_{\rho}\left(-\lambda_{k} \tau^{\rho}\right) \xi^{\rho} E_{\rho, \rho+1}\left(-\lambda_{k} \xi^{\rho}\right)}\right|^{2}\left|\varphi_{k}\right|^{2}=\sum_{k=1}^{j} \frac{\left|\varphi_{k}\right|^{2}}{\left|\xi^{\rho} E_{\rho, \rho+1}\left(-\lambda_{k} \xi^{\rho}\right)\right|^{2}} .
$$

By virtue of (52),

$$
I_{2, j} \leq \sum_{k=1}^{j} \frac{\lambda_{k}^{2}\left|\varphi_{k}\right|^{2}}{\left(1+O\left(\left(-\lambda_{k} \xi^{\rho}\right)^{-1}\right)\right)^{2}} \leq C \sum_{k=1}^{j} \lambda_{k}^{2}\left|\varphi_{k}\right|^{2} \leq C\|\varphi\|_{1}^{2} .
$$

Thus, if $\varphi, \Psi \in D(A)$, then from estimates of $I_{i, j}$ and (51) we obtain $f \in H$. 
After finding the unknown function $f \in H$, the fulfilment of the conditions of Definition 2 for function $u(t)$, defined by series (49) is proved in exactly the same way as with Corollary 1 and Theorem 2.

Uniqueness. Suppose we have two solutions: $\left\{u_{1}(t), f_{1}\right\}$ and $\left\{u_{2}(t), f_{2}\right\}$. It is required to prove $u(t) \equiv u_{1}(t)-u_{2}(t) \equiv 0$ and $f \equiv f_{1}-f_{2}=0$. Since the problem is linear, to determine $u(t)$ and $f$ we have the problem:

$$
\begin{gathered}
D_{t}^{\rho} u(t)+A u(t)=f, \quad t>0 \\
u(\xi)=\alpha u(0), \quad 0<\xi \leq T, \\
u(\tau)=0, \quad(\tau \neq \xi) .
\end{gathered}
$$

where $\xi$-fixed point.

Let $u(t)$ be a solution to this problem and $u_{k}(t)=\left(u(t), v_{k}\right)$. Then, by virtue of Equation (53) and the self-adjointness of operator $A$,

$$
\begin{gathered}
\partial_{t}^{\rho} u_{k}(t)=\left(\partial_{t}^{\rho} u(t), v_{k}\right)=-\left(A u(t), v_{k}\right)+\left(f, v_{k}\right)=-\left(u(t), A v_{k}\right)+\left(f, v_{k}\right)= \\
-\left(u(t), \lambda_{k} v_{k}\right)+f_{k}=-\lambda_{k}\left(u(t), v_{k}\right)+f_{k}=-\lambda_{k} u_{k}(t)+f_{k}, \quad t>0 .
\end{gathered}
$$

Thus, taking into account (54) and (55), we have the following problem

$$
\partial_{t}^{\rho} u_{k}(t)+\lambda_{k} u_{k}(t)=f_{k}, \quad t>0 ; \quad u_{k}(\xi)=\alpha u_{k}(0), \quad u_{k}(\tau)=0 .
$$

Suppose that $f_{k}$ is known and use the nonlocal condition to obtain (see, e.g., [20], p. 174)

$$
u_{k}(t)=\frac{f_{k} \xi^{\rho} E_{\rho, \rho+1}\left(-\lambda_{k} \xi^{\rho}\right)}{\alpha-E_{\rho}\left(-\lambda_{k} \xi^{\rho}\right)} E_{\rho}\left(-\lambda_{k} t^{\rho}\right)+f_{k} t^{\rho} E_{\rho, \rho+1}\left(-\lambda_{k} t^{\rho}\right) .
$$

Now apply $u_{k}(\tau)=0$ to obtain

$$
f_{k}\left[\xi^{\rho} E_{\rho, \rho+1}\left(-\lambda_{k} \xi^{\rho}\right) E_{\rho}\left(-\lambda_{k} \tau^{\rho}\right)+\tau^{\rho} E_{\rho, \rho+1}\left(-\lambda_{k} \tau^{\rho}\right)\left(\alpha-E_{\rho}\left(-\lambda_{k} \xi^{\rho}\right)\right)\right]=0 .
$$

Note, that if $\alpha \geq 1$ and $t>0$ then, $\alpha-E_{\rho}\left(-\lambda_{k} t^{\rho}\right)>0, E_{\rho}\left(-\lambda_{k} t^{\rho}\right)>0$ and $E_{\rho, \rho+1}\left(-\lambda_{k} t^{\rho}\right)>0$. Therefore, for all $k$ one has $f_{k}=0$. Hence, from the completeness of the system of eigenfunctions $\left\{v_{k}\right\}$, we finally obtain $f=0$ and $u(t) \equiv 0$, as required. The uniqueness is proved.

In Theorem 3, condition $E_{\rho}\left(-\lambda_{k} \xi^{\rho}\right) \neq \alpha$ for all $k$, ensured the uniqueness of the solution and no orthogonality conditions were required for its existence. In this inverse problem, the same condition does not guarantee the uniqueness of the solution. The reason is that at $\alpha<1$ the bracket in (57) can vanish for some $k_{0}$, and these numbers $k_{0}$ depend on $\alpha$ and the location of points $\xi$ and $\tau$. For the existence of an unknown function $f$ in this case, it is necessary to require the orthogonality conditions $\left(\varphi, v_{k_{0}}\right)=0$ and $\left(\Psi, v_{k_{0}}\right)=0$. Finding out the dependence of $k_{0}$ on these parameters is the subject of a separate paper. This is the reason why only case $\alpha \geq 1$ is considered in Theorem 4 .

On the other hand, in the next two theorems the condition $E_{\rho}\left(-\lambda_{k} \xi^{\rho}\right) \neq \alpha$ for all $k$ ensures the unique solvability of the problems under study. If $E_{\rho}\left(-\lambda_{k} \xi^{\rho}\right)=\alpha$ for some $k$, then, just as above (see the proof of Theorem 3), there is no uniqueness, and to ensure the existence of the solution, it is necessary to require the fulfillment of certain orthogonality conditions.

\section{The Inverse Problem of Determining the Boundary Function $\varphi$}

Consider the problem (2) and assume that, together with function $u(t)$, function $\varphi$ in the nonlocal condition $u(\xi)=\alpha u(0)+\varphi$ is also unknown. To solve this inverse problem, we 
need an additional condition, and as such we again take the condition that was used in the previous inverse problem:

$$
u(\tau)=\Psi, \quad 0<\tau \leq T, \quad \tau \neq \xi
$$

If $\tau=\xi$, then the nonlocal condition $u(\xi)=\alpha u(0)+\varphi$ coincides with the Cauchy condition $u(0)=\varphi_{1}$ (see (4)) and we have the inverse problem, considered in [2-4].

Definition 3. A pair $\{u(t), \varphi\}$ of function $u(t) \in C([0, T] ; H)$ and $\varphi \in H$ with the properties $D_{t}^{\rho} u(t), A u(t) \in C((0, T) ; H)$ and satisfying conditions (2), (58) are called the solution of the inverse problem (2), (58).

As noted at the end of Section 4, an additional condition is imposed on $\alpha$, which simplifies the proof of the uniqueness of the solution.

Theorem 5. Let $\Psi \in D(A), f \in C\left([0, T] ; D\left(A^{\varepsilon}\right)\right)$ for some $\varepsilon \in(0,1)$ and $E_{\rho}\left(-\lambda_{k} \xi^{\rho}\right) \neq \alpha$ for all $k$. Then the inverse problem (2), (58) has a unique solution $\{u(t), \varphi\}$ and this solution has the form

$$
\begin{aligned}
& \varphi=\sum_{k=1}^{\infty}\left[\frac{E_{\rho}\left(-\lambda_{k} \xi^{\rho}\right)-\alpha}{E_{\rho}\left(-\lambda_{k} \tau^{\rho}\right)}\left[\Psi_{k}-\omega_{k}(\tau)\right]+\omega_{k}(\xi)\right] v_{k}, \\
& u(t)=\sum_{k=1}^{\infty}\left[\frac{\varphi_{k}-\omega_{k}(\xi)}{E_{\rho}\left(-\lambda_{k} \xi^{\rho}\right)-\alpha} E_{\rho}\left(-\lambda_{k} t^{\rho}\right)+\omega_{k}(t)\right] v_{k},
\end{aligned}
$$

where

$$
\omega_{k}(t)=\int_{0}^{t} \eta^{\rho-1} E_{\rho, \rho}\left(-\lambda_{k} \eta^{\rho}\right) f_{k}(t-\eta) d \eta
$$

Proof of Theorem 5. Existence. If $\varphi$ is known, then the solution of problem (3) has the form (60) (see Theorem 3). Condition (58) implies:

$$
u(\tau)=\sum_{k=1}^{\infty}\left[\frac{\varphi_{k}-\omega_{k}(\xi)}{E_{\rho}\left(-\lambda_{k} \xi^{\rho}\right)-\alpha} E_{\rho}\left(-\lambda_{k} \tau^{\rho}\right)+\omega_{k}(\tau)\right] v_{k}=\Psi .
$$

Let us expand function $\Psi \in H$ in a Fourier series in system $\left\{v_{k}\right\}$. Then

$$
\frac{\varphi_{k}-\omega_{k}(\xi)}{E_{\rho}\left(-\lambda_{k} \xi^{\rho}\right)-\alpha} E_{\rho}\left(-\lambda_{k} \tau^{\rho}\right)+\omega_{k}(\tau)=\Psi_{k}, k \geq 1
$$

or

$$
\varphi_{k}=\frac{E_{\rho}\left(-\lambda_{k} \xi^{\rho}\right)-\alpha}{E_{\rho}\left(-\lambda_{k} \tau^{\rho}\right)}\left[\Psi_{k}-\omega_{k}(\tau)\right]+\omega_{k}(\xi)
$$

Therefore, equality (59) is formally established. It remains to prove that $\varphi \in H$. Let $\Phi_{j}$ be the partial sum of series (59). Then due to Parseval's equality

$$
\begin{gathered}
|| \Phi_{j} \|^{2}=\sum_{k=1}^{j}\left|\frac{E_{\rho}\left(-\lambda_{k} \xi^{\rho}\right)-\alpha}{E_{\rho}\left(-\lambda_{k} \tau^{\rho}\right)}\left[\Psi_{k}-\omega_{k}(\tau)\right]+\omega_{k}(\xi)\right|^{2} \leq \\
\leq 3 \sum_{k=1}^{j}\left[\left|\frac{E_{\rho}\left(-\lambda_{k} \xi^{\rho}\right)-\alpha}{E_{\rho}\left(-\lambda_{k} \tau^{\rho}\right)}\right|^{2}\left[\left|\Psi_{k}\right|^{2}+\left|\omega_{k}(\tau)\right|^{2}\right]+\left|\omega_{k}(\xi)\right|^{2}\right] \equiv \Phi_{j}^{1}+\Phi_{j}^{2}+\Phi_{j}^{3} .
\end{gathered}
$$

Since $\left|E_{\rho}\left(-\lambda_{k} \xi^{\rho}\right)-\alpha\right| \leq C$, then by virtue of the asymptotic estimate (8), we obtain

$$
\Phi_{j}^{1} \leq C \sum_{k=1}^{j} \frac{\lambda_{k}^{2} \tau^{2 \rho} \Gamma^{2}(1-\rho)}{\left(1+O\left(\left(-\lambda_{k} \tau^{\rho}\right)^{-1}\right)\right)^{2}}\left|\Psi_{k}\right|^{2} \leq C \sum_{k=1}^{j} \lambda_{k}^{2}\left|\Psi_{k}\right|^{2} \leq C\|\Psi\|_{1}^{2} .
$$


Similarly, by virtue of the estimate (11) and the definition of $\omega_{k}$, we have

$$
\begin{aligned}
\Phi_{j}^{2} \leq & C \sum_{k=1}^{j} \frac{\lambda_{k}^{2} \tau^{2 \rho} \Gamma^{2}(1-\rho)}{\left(1+O\left(\left(-\lambda_{k} \tau^{\rho}\right)^{-1}\right)\right)^{2}}\left|\int_{0}^{\tau} \eta^{\rho-1} E_{\rho, \rho}\left(-\lambda_{k} \eta^{\rho}\right) f_{k}(\tau-\eta) d \eta\right|^{2} \leq \\
& \leq \sum_{k=1}^{j} \frac{C_{\varepsilon} \lambda_{k}^{2}}{\left(1+O\left(\left(-\lambda_{k} \tau^{\rho}\right)^{-1}\right)\right)^{2}}\left|\int_{0}^{\tau} \eta^{\varepsilon \rho-1} \lambda_{k}^{\varepsilon-1}\right| f_{k}(\tau-\eta)|d \eta|^{2} \leq \\
& \leq C_{\varepsilon}\left[\int_{0}^{\tau} \eta^{\varepsilon \rho-1}\left(\sum_{k=1}^{j}\left|\lambda_{k}^{\varepsilon} f_{k}(\tau-\eta)\right|^{2}\right)^{\frac{1}{2}} d \eta\right]^{2} \leq C_{\varepsilon} \max _{t \in[0, T]}\|f\|_{\varepsilon}^{2} .
\end{aligned}
$$

For the sum $\Phi_{j}^{3}$ one has

$$
\begin{gathered}
\Phi_{j}^{3} \leq \sum_{k=1}^{j}\left|\int_{0}^{\xi} \eta^{\rho-1} E_{\rho, \rho}\left(-\lambda_{k} \eta^{\rho}\right) f_{k}(\xi-\eta) d \eta\right|^{2} \leq \\
\leq C\left[\int_{0}^{\zeta} \eta^{\rho-1}\left(\sum_{k=1}^{j}\left|f_{k}(\xi-\eta)\right|^{2}\right)^{\frac{1}{2}} d \eta\right]^{2} \leq C \max _{t \in[0, T]}\|f\|^{2} .
\end{gathered}
$$

Thus, it is shown that $\varphi \in H$ (see (61)).

Fulfillment of the conditions of Definition 3 for function $u(t)$, defined by the series (60) is proved in exactly the same way as Theorem 3.

Uniqueness. Let us prove that if $\{u(t), \varphi\}$ is a solution to the homogeneous problem:

$$
\begin{aligned}
& D_{t}^{\rho} u(t)+A u(t)=0, \quad t>0 \\
& u(\xi)=\alpha u(0)+\varphi, \quad 0<\xi \leq T, \\
& u(\tau)=0, \quad 0<\tau \leq T, \quad \tau \neq \xi
\end{aligned}
$$

then $u(t) \equiv 0$ and $\varphi=0$.

Let $u(t)$ be a solution to this problem and let $u_{k}(t)=\left(u(t), v_{k}\right)$. Then

$$
D_{t}^{\rho} u_{k}(t)+\lambda_{k} u_{k}(t)=0, \quad t>0 ; \quad u_{k}(\xi)=\alpha u_{k}(0)+\varphi_{k} .
$$

The solution to this problem has the form (this is the same problem as (23))

$$
u_{k}(t)=\frac{E_{\rho}\left(-\lambda_{k} t^{\rho}\right)}{E_{\rho}\left(-\lambda_{k} \xi^{\rho}\right)-\alpha} \varphi_{k} .
$$

Condition (64) implies

$$
u_{k}(\tau)=\frac{E_{\rho}\left(-\lambda_{k} \tau^{\rho}\right)}{E_{\rho}\left(-\lambda_{k} \xi^{\rho}\right)-\alpha} \varphi_{k}=0 .
$$

Since $E_{\rho}\left(-\lambda_{k} \xi^{\rho}\right) \neq \alpha$ for all $k \geq 1$, then by virtue of the properties of the Mittag-Leffler functions (see Proposition 2) we have $\varphi_{k}=0$ for all $k$. This, in turn, means $u_{k}(t) \equiv 0$ for all $k$. Therefore, due to the completeness of the system of eigenfunctions $\left\{v_{k}\right\}$, we have $\varphi=0$ and $u(t) \equiv 0$, as required.

Theorem 5 is completely proved. 
Remark 1. If $f$ does not depend on $t$, then the statement of Theorem 5 is true for all $f \in H$, and $\Psi \in D(A)$.

Using Corollary 1, this statement can be proved in the same way as above.

\section{Well-Posedness of Problem (3)}

In the case of fractional Riemann-Liouville derivatives, we consider only the forward problem for the homogeneous subdiffusion equation. The inhomogeneous equations and inverse problems considered above are studied in exactly the same way as in the case of the Caputo derivatives.

Consider the forward problem:

$$
\left\{\begin{array}{l}
\partial_{t}^{\rho} u(t)+A u(t)=0, \quad 0<t \leq T \\
\left.\partial_{t}^{\rho-1} u(t)\right|_{t=\xi}=\alpha \lim _{t \rightarrow 0} \partial_{t}^{\rho-1} u(t)+\phi, \quad 0<\xi \leq T
\end{array}\right.
$$

where $\phi \in H$ and the number $\alpha$ are given.

As was mentioned above (see Section 4) we will only consider the case $E_{\rho}\left(-\lambda_{k} \xi^{\rho}\right) \neq \alpha$ for all $k \geq 1$.

Theorem 6. Let $\phi \in H$ and $E_{\rho}\left(-\lambda_{k} \xi^{\rho}\right) \neq \alpha$ for all $k \geq 1$. Then problem (65) has a unique solution and this solution has the form

$$
u(t)=\sum_{k=1}^{\infty} \frac{\phi_{k}}{E_{\rho}\left(-\lambda_{k} \xi^{\rho}\right)-\alpha} t^{\rho-1} E_{\rho, \rho}\left(-\lambda_{k} t^{\rho}\right) v_{k} .
$$

Moreover, there is a constant $C_{\xi}>0$ such that the following coercive type inequality holds:

$$
\left\|\partial_{t}^{\rho} u(t)\right\|_{1}^{2}+\|u(t)\|_{2}^{2} \leq C_{\xi} t^{-2 \rho-2}\|\phi\|^{2}, \quad 0<t \leq T .
$$

Proof. As in the case of problem (2), we will seek a solution to problem (65) in the form of a series: $\sum_{k=1}^{\infty} T_{k}(t) v_{k}$, where $T_{k}(t)$ is a solution to the problem:

$$
\begin{gathered}
\partial_{t}^{\rho} T_{k}(t)+\lambda_{k} T_{k}(t)=0, \quad 0<t \leq T ; \\
\left.\partial_{t}^{\rho-1} T_{k}(t)\right|_{t=\xi}=\alpha \lim _{t \rightarrow 0} \partial_{t}^{\rho-1} T_{k}(t)+\phi_{k}, \quad 0<\xi \leq T .
\end{gathered}
$$

Let us denote $\lim _{t \rightarrow 0} \partial_{t}^{\rho-1} T_{k}(t)=b_{k}$. Then the unique solution to the differential Equation (68) with this initial condition has the form $T_{k}(t)=b_{k} t^{\rho-1} E_{\rho, \rho}\left(-\lambda_{k} t^{\rho}\right.$ ) (see, e.g., [20], p. 173, [52]).

Proposition 5 implies

$$
\left.\partial_{t}^{\rho-1} T_{k}(t)\right|_{t=\xi}=b_{k} E_{\rho}\left(-\lambda_{k} \xi^{\rho}\right)
$$

Then from the nonlocal conditions (69) we find the unknown numbers $b_{k}$ :

$$
b_{k}=\frac{\phi_{k}}{E_{\rho}\left(-\lambda_{k} \xi^{\rho}\right)-\alpha} \text {. }
$$

Hence, function $u(t)$ defined by series (66) is a formal solution to problem (65). 
Now let us show that series (66) is indeed a solution. To do this we denote by $S_{j}(t)$ the partial sum of series (66). Then

$$
A^{2} S_{j}(t)=\sum_{k=1}^{j} \lambda_{k}^{2} \frac{\phi_{k}}{E_{\rho}\left(-\lambda_{k} \xi^{\rho}\right)-\alpha} t^{\rho-1} E_{\rho, \rho}\left(-\lambda_{k} t^{\rho}\right) v_{k}
$$

Due to the Parseval equality we may write

$$
\left\|A^{2} S_{j}(t)\right\|^{2}=\sum_{k=1}^{j}\left|\lambda_{k}^{2} \frac{\phi_{k}}{E_{\rho}\left(-\lambda_{k} \xi^{\rho}\right)-\alpha} t^{\rho-1} E_{\rho, \rho}\left(-\lambda_{k} t^{\rho}\right)\right|^{2} .
$$

Estimate (10) of function $E_{\rho, \rho}(-t)$ implies (note, $E_{\rho}\left(-\lambda_{k} \xi^{\rho}\right) \neq \alpha$ )

$$
\left\|A^{2} S_{j}(t)\right\|^{2} \leq \frac{C t^{2 \rho-2}}{\left|\alpha-E_{\rho}\left(-\lambda_{1} \xi^{\rho}\right)\right|^{2}} \sum_{k=1}^{j} \lambda_{k}^{4}\left|\frac{\phi_{k}}{1+\lambda_{k}^{2} t^{2 \rho}}\right|^{2} \leq C_{\xi} t^{-2 \rho-2} \sum_{k=1}^{j}\left|\phi_{k}\right|^{2} \leq C_{\xi} t^{-2 \rho-2}\|\phi\|^{2} .
$$

Thus $A^{2} u(t) \in C((0, T] ; H)$ (and even more so $A u(t) \in C((0, T] ; H)$ ). Since $A \partial_{t}^{\rho} u(t)=-A^{2} u(t)$, then from the above estimate we have

$$
\left\|\partial_{t}^{\rho} u(t)\right\|_{1}^{2} \leq C_{\xi} t^{-2 \rho-2}\|\phi\|^{2}
$$

Thus, (66) is a solution to problem (65). The coercivity inequality follows from the last two estimates.

The uniqueness is proved in the same way as in the proof of Theorem 2.

Remark 2. Having considered problem (65), we would like to draw the reader's attention to the fact that although in the case of the Riemann-Liouville derivatives function $T_{k}(t)$ is determined using Mittag-Leffler function with two parameters, but thanks to Proposition 5 , the coefficients $b_{k}$ are determined in exactly the same way as in the case of the Caputo derivatives. It is this fact that allows us to apply the same reasoning as above to solve the problem for an inhomogeneous equation with Riemann-Liouville derivatives and inverse problems.

\section{Conclusions}

We have considered the following three problems: (1) the nonlocal in time forward problems (2) and (3); (2) the inverse source problem (46) with additional condition (47); (3) the inverse problem of determining the boundary function $\varphi$. To the best of the authors' knowledge, forward problems (2) and (3) with such nonlocal conditions for differential equations with fractional derivatives have not been discussed before. In the study of forward problems, we determined the influence of parameter $\alpha$ on the correctness of the problems under study. It should be noted, as proved above, for some values of $\alpha$ there is no uniqueness of the solution, and to ensure the existence of a solution, it is necessary to require orthogonality conditions for the given functions $\varphi$ and $f$. From the proofs of Theorems 4 and 5, it can be seen that the same values of the parameter $\alpha$ violate the uniqueness of solutions to inverse problems.

In [45] nonlocal in time (forward and space-dependent inverse source) problems for a so-called generalized time fractional diffusion equation were considered. The fractional derivative used in [45] is the Hilfer derivative, which is a generalization of the well-known Riemann-Liouville and Caputo fractional derivatives. Indeed, the generalized fractional derivative interpolates the Riemann-Liouville and Caputo fractional derivatives. The results presented in this paper can be extended by considering the Hilfer derivative.

Author Contributions: Conceptualization, R.A. and Y.F. All authors have read and agreed to the published version of the manuscript.

Funding: This research received no external funding. 
Institutional Review Board Statement: Not applicable.

Informed Consent Statement: Not applicable.

Data Availability Statement: Not applicable.

Acknowledgments: The authors are grateful to A. O. Ashyralyev for posing the problem and they convey thanks to Sh. A. Alimov for discussions of these results. We also want to express our special thanks to the anonymous journal reviewers for their comments, which have greatly improved the content of this article.

Conflicts of Interest: The authors declare no conflict of interest.

\section{References}

1. Lizama, C. Abstract Linear Fractional Evolution Equations. In Handbook of Fractional Calculus with Applications V. 2; Marchado, J.A.T., Ed.; De Gruyter: Berlin, Germany; Munich, Germany; Boston, MA, USA, 2019; pp. 465-497. [CrossRef]

2. Liu, J.; Yamamoto, M. A backward problem for the time-fractional diffusion equation. Appl. Anal. 2010, 89, 1769-1788. [CrossRef]

3. Sakamoto, K.; Yamamoto, M. Initial value/boundary value problems for fractional diffusion-wave equations and applications to some inverse problems. J. Math. Anal. Appl. 2011, 382, 426-447. [CrossRef]

4. Floridia, G.; Li, Z.; Yamamoto, M. Well-posedness for the backward problems in time for general time-fractional difussion equation. Rend. Lincei Mat. Appl. 2020, 31, 593-610.

5. Alimov, S.A.; Ashurov, R.R. On the Backward Problems in Time For Time-Fractional Subdiffusion Equations. Available online: https:/ / www.researchgate.net/publication/351575279 (accessed on 1 May 2021).

6. Kabanikhin, S.I. Inverse and Ill-Posed Problems. In Theory and Applications; De Gruyter: Berlin, Germany; Munich, Germany; Boston, MA, USA, 2011. Available online: https:/ / www.twirpx.com/file/2069361/ (accessed on 1 May 2021).

7. Tuan, N.H.; Huynh, L.N.; Ngoc, T.B.; Zhou, Y. On a backward problem for nonlinear fractional diffusion equations. Appl. Math. Lett. 2019, 92, 76-84. [CrossRef]

8. Tuan, N.H.; Long, L.D.; Tatar, S. Tikhonov regularization method for a backward problem for the inhomogeneous time-fractional diffusion equation. Appl. Anal. 2018, 97, 842-863. [CrossRef]

9. Wang, L.; Liu, J. Total variation regularization for a backward timefractional diffusion problem. Inverse Probl. 2013, 29 , 115-131. [CrossRef]

10. Umarov, S.R. Introduction to Fractional and Pseudo-Differential Equations with Singular Symbols; Springer: Berlin/Heidelberg, Germany, 2015. doi: 10.1007/978-3-319-20771-1. [CrossRef]

11. Pao, C.V. Reaction diffusion equations with nonlocal boundary and nonlocal initial conditions. J. Math. Anal. Appl. 1995, 195, 702-718. [CrossRef]

12. Tuan, N.H.; Triet, N.A.; Luc, N.H.; Phuong, N.D. On a time fractional diffusion with nonlocal in time conditions. Adv. Differ. Equ. 2021, 204, 1-14. [CrossRef]

13. Ashyralyev, A.O.; Sobolevskii, P.E. Coercive stability of a multidimensional difference elliptic equation of $2 \mathrm{~m}$-th order with variable coefficients. In Investigations in the Theory of Differential Equations (Russian); Minvuz Turkmen. SSR: Ashkhabad, Turkmenistan, 1987. pp. 31-43. Available online: https:// mathscinet.ams.org/mathscinet-getitem? mr=1+009+419 (accessed on 1 May 2021).

14. Ashyralyev, A.O.; Hanalyev, A.; Sobolevskii, P.E. Coercive solvability of nonlocal boundary value problem for parabolic equations. Abstr. Appl. Anal. 2001, 6, 53-61. [CrossRef]

15. Krein, S.G. Linear Differential Equations in a Banach Space; Nauka: Moscow, Russia, 1966. (In Russian)

16. Prilepko, A.I.; Orlovsky, D.G.; Vasin, I.A. Methods for Solving Inverse Problems in Mathematical Physics; Marcel Dekkers: New York, NY, USA, 2000.

17. Yuldashev, T.K.; Kadirkulov, B.J. Inverse Problem for a Partial Differential Equation with Gerasimova-Caputo-Type Operator and Degeneration. Fractal Fract. 2021, 5, 58. [CrossRef]

18. Pskhu, A.V. Fractional Partial Differential Equations, Nauka, M., Ed.; 2005. Available online: https://www.elibrary.ru/item.asp? $\mathrm{id}=19448655$ (accessed on 1 May 2021). (In Russian)

19. Dzherbashian, M.M. Integral Transforms and Representation of Functions in the Complex Domain; Nauka, M., Ed.; 1966. Available online: https:/ / www.twirpx.com/file/1753304/ (accessed on 1 May 2021). (In Russian)

20. Gorenflo, R.; Kilbas, A.A.; Mainardi, F.; Rogozin, S.V. Mittag-Leffler Functions, Related Topics and Applications; Springer: Berlin/Heidelberg, Germany, 2014. doi: 10.1007/978-3-662-61550-8. [CrossRef]

21. Ashurov, R.; Cabada, A.; Turmetov, B. Operator method for construction of Solutions of Linear Fractional Differential Equations with Constant coefficients. Fract. Calc. Appl. Anal. 2016, 1, 229-252. [CrossRef]

22. Il'in, V.A. On the solvability of mixed problems for hyperbolic and parabolic equations. Russ. Math. Surv. 1960, 15, 85-142 [CrossRef]

23. Liu, Y.; Li, Z.; Yamamoto, M. Inverse Problems of Determining Sources of the Fractional Partial Differential Equations. In Handbook of Fractional Calculus with Applications V. 2; Marchado, J.A.T., Ed.; De Gruyter: Berlin, Germany; Munich, Germany; Boston, MA, USA, 2019; pp. 411-430. [CrossRef] 
24. Ashyralyev, A.; Urun, M. Time-dependent source identification problem for the Schrodinger equation with nonlocal boundary conditions. AIP Conf. Proc. 2019, 2183, 070016.

25. Ashyralyev, A.; Urun, M. On the Crank-Nicolson difference scheme for the time-dependent source identification problem. Bull. Karaganda Univ. Math. 2021, 102, 35-44. [CrossRef]

26. Ashyralyev, A.; Urun, M. Time-dependent source identification Schrodinger type problem. Int. J. Appl. Math. 2021, 34, 297-310. http:/ /dx.doi.org/10.12732/ijam.v34i2.7 [CrossRef]

27. Niu, P.; Helin, T.; Zhang, Z. An inverse random source problem in a stochastic fractional diffusion equation. Inverse Probl. 2020, 36, 045002. [CrossRef]

28. Slodichka, M.; Sishskova, K.; Bockstal, V. Uniqueness for an inverse source problem of determining a space dependent source in a time-fractional diffusion equation. Appl. Math. Lett. 2019, 91, 15-21. [CrossRef]

29. Slodichka, M. Uniqueness for an inverse source problem of determining a space-dependent source in a non-autonomous time-fractional diffusion equation. Frac. Calc. Appl. Anal. 2020, 23, 1703-1711. [CrossRef]

30. Zhang, Y.; Xu, X. Inverse scource problem for a fractional differential equations. Inverse Prob. 2011, 27, 31-42. [CrossRef]

31. Furati, K.M.; Iyiola, O.S.; Kirane, M. An inverse problem for a generalized fractional diffusion. Appl. Math. Comput. 2014, 249, 24-31. [CrossRef]

32. Ismailov, M.I.; Cicek, M. Inverse source problem for a time-fractional diffusion equation with nonlocal boundary conditions Appl. Math. Model. 2016, 40, 4891-4899. [CrossRef]

33. Kirane, M.; Malik, A.S. Determination of an unknown source term and the temperature distribution for the linear heat equation involving fractional derivative in time. Appl. Math. Comput. 2011, 218, 163-170. [CrossRef]

34. Kirane, M.; Samet, B.; Torebek, B.T. Determination of an unknown source term and the temperature distribution for the subdiffusion equation at the initial and final data. Electron. J. Differ. Equ. 2017, 217, 1-13.

35. Nguyen, H.T.; Le, D.L.; Nguyen, V.T. Regularized solution of an inverse source problem for a time fractional diffusion equation. Appl. Math. Model. 2016, 40, 8244-8264. [CrossRef]

36. Torebek, B.T.; Tapdigoglu, R. Some inverse problems for the nonlocal heat equation with Caputo fractional derivative. Math Methods Appl. Sci. 2017, 40, 6468-6479. [CrossRef]

37. Li, Z.; Liu, Y.; Yamamoto, M. Initial-boundary value problem for multi-term time-fractional diffusion equation with positive constant coefficients. Appl. Math. Comput. 2015, 257, 381-397. [CrossRef]

38. Rundell, W.; Zhang, Z. Recovering an unknown source in a fractional diffusion problem. J. Comput. Phys. 2018, 368, 299-314. [CrossRef]

39. Asl, N.A.; Rostamy, D. Identifying an unknown time-dependent boundary source ib time-fractional diffusion equation with a non-local boundary condition. J. Comput. Appl. Math. 2019, 335, 36-50. [CrossRef]

40. Sun, L.; Zhang, Y.; Wei, T. Recovering the time-dependent potential function in a multi-term time-fractional diffusion equation. Appl. Numer. Math. 2019, 135, 228-245. [CrossRef]

41. Malik, S.A.; Aziz, S. An inverse source problem for a two parameter anomalous diffusion equation with nonlocal boundary conditions. Comput. Math. Appl. 2017, 3, 7-19. [CrossRef]

42. Ruzhansky, M.; Tokmagambetov, N.; Torebek, B.T. Inverse source problems for positive operators. I: Hypoelliptic diffusion and subdiffusion equations. J. Inverse Ill-Possed Probl. 2019, 27, 891-911. [CrossRef]

43. Ashurov, R.; Muhiddinova, O. Inverse problem of determining the heat source density for the subdiffusion equation. Differ. Equ. 2020, 56, 1550-1563. [CrossRef]

44. Ashurov, R.; Muhiddinova, O. Initial-boundary value problem for a time-fractional subdiffusion equation with an arbitrary elliptic differential operator. Lobachevskii J. Math. 2021, 42, 517-525. [CrossRef]

45. Shuang, Z.; Saima, R.; Asia, R.; Khadija, K.; Abdullah, M.A. Initial boundary value problems for a multi-term time fractional diffusion equation with generalized fractional derivatives in time. AIMS Math. 2021, 6, 12114-12132. [CrossRef]

46. Kirane, M.; Malik, S.A.; Al-Gwaiz, M.A. An inverse source problem for a two dimensional time fractional diffusion equation with nonlocal boundary conditions. Math. Meth. Appl. Sci. 2012, 36, 1056-1069. [CrossRef]

47. Muhammad, A.; Salman, A.M. An inverse problem for a family of time fractional diffusion equations. Inverse Probl. Sci. Eng. 2016, 25, 1299-1322. [CrossRef]

48. Ashurov, R.; Fayziev, Y. Determination of Fractional Order and Source Term in a Fractional Subdiffusion Equation. Available online: https:/ / www.researchgate.net/publication/354997348 (accessed on 1 October 2021).

49. Hilfer, R.; Luchko, Y.; Tomovski, Z. Operational method for solution of the fractional differential equations with the generalized Riemann-Liouville fractional derivatives. Frac. Calculus Appl. Anal. 2009, 12, 299-318.

50. Ashurov, R.; Fayziev, Y. Uniqueness and existence for inverse problem of determining an order of time-fractional derivative of subdiffusion equation. Lobachevskii J. Math. 2021, 42, 508-516. [CrossRef]

51. Ashurov, R.; Fayziev, Y. Inverse problem for determining the order of the fractional derivative in the wave equation. Math. Notes 2021, 110, 842-852. [CrossRef]

52. Ashurov, R.; Fayziev, Y. On construction of solutions of linear fractional differentional equations with constant coefficients and the fractional derivatives. Uzb. Math. J. 2017, 3, 3-21. (In Russian) 